\title{
Rechtsgeschichte
}

\section{Jan Schröder}

\section{Zivilistisches und publizistisches Denken um 1900. Zwei Beispiele}


I M. Stolleis, Geschichte des öffentlichen Rechts in Deutschland. 3. Band, Staats- und Verwaltungsrechtswissenschaft in Republik und Diktatur I9I4-I945, München I999, 6.

2 Zur Geschichte der Gewohnheitsrechtstheorie H. KRAUSE, G. KöBLER, Gewohnheitsrecht, in: HRG, 2. Aufl., II (IO. Lieferung), 2009, 364-375; J. SCHRÖDER, Recht als Wissenschaft. Geschichte der juristischen Methode vom Humanismus bis zur historischen Schule (I 500-I 850), München 200I, I4 f., I05-I08, I94-I98. Zum Folgenden vor allem J. ScHRöDER. Zur Theorie des Gewohnheitsrechts zwischen I 850 und I930, in: Usus modernus pandectarum, hg. von H.-P. HAFERKAMP, T. REPGEN (2007), wieder in DERS., Rechtswissenschaft in der Neuzeit, Tübingen 20IO, 547-568.

3 Gegen das Erfordernis staatlicher Gestattung sind u. a. E. ZITELMANN, Gewohnheitsrecht und Irrtum, in: AcP 66 (I883) 323-468 (36I ff.); F. EISELE, Unverbindlicher Gesetzesinhalt, Freiburg I 88 5, I9 ff.; C. F. v. Gerber, System des deutschen Privatrechts, I7. Aufl. von K. Cosack, Jena I895, 29 f. mit Anm. 5; O. GierKE, Deutsches Privatrecht, I, Leipzig I 895, I63 (gesetzliches Verbot ist aber nicht wirkungslos, I74); P. Oertmann, Volksrecht und Gesetzesrecht, Dresden I 898, 33 f.; C. Crome, System des Deutschen Bürgerlichen Rechts, I, Tübingen I900, 84-86, 89; wohl auch M. RüMelin, Das neue schweizerische Zivilgesetzbuch und seine Bedeutung für uns, Tübingen I908, 35 ; A. v. TuHR, Der Allgemeine Teil des Deutschen Bürgerlichen Rechts, I. Band, Leipzig I9IO, Iof.; P. KRÜCKMANN, Einführung in das Recht, Tübingen I9I2, 82; G. PlancK, Kommentar zum Bürgerlichen Gesetzbuch nebst Einführungsgesetz, I. Band, 4. Aufl. Berlin I9I3, Einleitung, XXXIX (bearb. von PlanCK und P. KNOKE); H. ReICHEL, Gesetz und Richterspruch, Zürich I9I 5, 93 f., 98, I3 I f.; O. Fischer, Einführung in die Wissenschaft von Recht und Staat. Für Gebildete aller Berufe, München I920, I 8; TH. STERnBerg,

\section{Zivilistisches und publizistisches Denken um I900. Zwei Beispiele}

Michael Stolleis hat in seiner fundamentalen Geschichte des öffentlichen Rechts die Entfaltung der öffentlichrechtlichen Wissenschaft geschildert und ihren Aufstieg zu einer dem Zivilrecht gleichwertigen Disziplin. Für das I9. und 20. Jahrhundert stellt er eine »Ausdifferenzierung der einzelnen Unterdisziplinen innerhalb der Juristischen Fakultäten « fest und das Aufkommen des »leidige(n) Problem(s) der Interdisziplinarität «. »Der rechtstheoretische Topos der `Einheit der Rechtsordnung « « scheine »im 20. Jahrhundert seine Kraft zu verlieren «. ${ }^{\mathbf{x}}$

Wie zutreffend diese Beobachtung ist, zeigt sich vor allem im Verhältnis von privatrechtlicher und öffentlichrechtlicher Wissenschaft. Zivilistik und Publizistik um I900 haben nicht nur, was selbstverständlich ist, ihre besonderen, voneinander unterschiedenen Fachgebiete, sondern kommen offenbar auch bei allgemein rechtstheoretischen, die ganze Rechtswissenschaft umfassenden Fragen manchmal zu ganz unterschiedlichen Lösungen. Es scheint eine spezifisch zivilistische und eine spezifisch publizistische Denkweise zu geben, die weit über die eigene Disziplin hinaus die Einstellung zu Grundfragen des Rechts prägt. Ich möchte das anhand der Diskussion über zwei rechtstheoretische Probleme, nämlich den Begriff des Gewohnheitsrechts (I.) und die Reichweite der Gesetzesinterpretation - am Beispiel des Redaktionsversehens (2.) zu zeigen versuchen.

\section{Der Begriff des Gewohnheitsrechts}

Während in der frühesten Neuzeit Gewohnheits- ${ }^{2}$ und Gesetzesrecht als gleichwertige Rechtsquellen galten, von denen die jeweils spätere die frühere aufheben konnte, unterwirft man das Gewohnheitsrecht im I8. Jahrhundert mehr und mehr dem Staat und dem Gesetz. Gewohnheitsrecht soll jetzt nur noch mit $\mathrm{Zu}$ stimmung des staatlichen Gesetzgebers entstehen können; gesetzesaufhebendes Gewohnheitsrecht ist so gut wie ausgeschlossen.

\footnotetext{
Einführung in die Rechtswissenschaft, 2. Aufl. Berlin, Leipzig I920, I6I f.; J. v. STAUDINGER, Kommentar zum Bürgerlichen Gesetzbuch und dem Einführungsgesetze, r. Band, 9. Aufl. München [u. a.] I925, Einleitung I3 (bearb. von Th. Loewenfeld, E. RiezLER); J. W. Hedemann, Einführung in die Rechtswissenschaft, 2. Aufl. Berlin, Leipzig 1927, 88; K. Cosack, H. Mitteis, Lehrbuch des Bürgerlichen Rechts, I. Band, 8. Aufl. Jena I927, I7; E. JUnG, Das sogenannte Gewohnheitsrecht
}

als Grundfrage der Rechtsquellenlehre, in: ARWPh 22 (1928/29) 227-259 (235, 24I); M. RÜMELIN, Die bindende Kraft des Gewohnheitsrechts und ihre Begründung, Tübingen I929, 35 .

4 E. v. MeIer, Die Rechtsbildung in Staat und Kirche, Berlin I86I, 23,83 ; M. SEYDEL, Bayerisches Staatsrecht, 3. Band, 2. unv. Abdruck, Freiburg i. Br. I888, 549; P. Laband, Das Staatsrecht des Deutschen Reiches, 4. Aufl., 2. Band, Tübingen, Leipzig I90I, \5I III, 69, II, 5. Aufl., 75 Anm. I 
Demgegenüber entdeckt im frühen I9. Jahrhundert die historische Schule das Gewohnheitsrecht wieder als ursprüngliche Rechtsquelle. Als »Volksrecht « und vor allem als »Juristenrecht « soll es mindestens den gleichen Rang wie das Gesetz und auch wieder gesetzesderogierende Kraft haben.

Der Rechtswissenschaft des späten I9. und frühen 20. Jahrhunderts standen also zwei Modelle zur Verfügung. Das Recht war entweder, wie in der historischen Schule, (auch) eine Schöpfung des Volkes oder, wie in der Theorie des I 8. Jahrhunderts, nur eine des Staates. Je nachdem, welches Modell man bevorzugte, wurde das Gewohnheitsrecht entweder autonom vom Volk (bzw. seinen Repräsentanten) hervorgebracht oder nur unter Kontrolle des Staates, der es gestatten musste (» Gestattungstheorie «). Es ist nun auffällig, dass die Zivilrechtler ganz überwiegend für die erste Lösung votieren, ${ }^{3}$ die Öffentlichrechtler, d. h. hier die Vertreter des Staatsund Verwaltungsrechts, dagegen durchweg für die zweite. ${ }^{4}$ Zwar verläuft die Grenze nicht ganz scharf zwischen Zivilisten und Publizisten. Während aber immerhin einige Zivilisten (und Strafrechtler) doch auch der Gestattungstheorie zuneigen, ${ }^{5}$ gibt es anscheinend überhaupt keinen Staats- oder Verwaltungsrechtler, der sich umgekehrt eine staatsfreie Entstehung des Gewohnheitsrechts vorstellen kann. Die einzige Konzession ist, dass einige Publizisten eine Anerkennung des Gewohnheitsrechts durch staatliche Rechtsanwendungsorgane ausreichen lassen wollen. ${ }^{6}$

Dass sich die Frage nicht mit juristischen Argumenten entscheiden ließ, wird im Laufe des frühen 20. Jahrhunderts deutlich. Nach Ansicht der (meisten) Zivilisten kann der Gesetzgeber das Gewohnheitsrecht so wenig verbieten »als Jemand im Stande ist, seiner Leibeslänge eine Elle zuzusetzen «. ${ }^{7}$ Das Gewohnheitsrecht sei die »ursprüngliche Erscheinungsform des Rechtes «, deshalb könne sein Geltungsgrund nicht wiederum ein anderer Rechtsgrund, also das staatliche Gesetz sein. ${ }^{8}$ Dagegen ergibt sich für die Publizisten schon »aus dem Begriff des Gesetzes «, dass Gewohnheitsrecht keine derogatorische Kraft haben kann und Staatsorgane nicht »einen anderen Willen als den ihres Staates zur Ausführung bringen « dürfen. ${ }^{9}$ Rechtsnormen können »als Bestandteile eines und desselben Rechtssystems nur dann angesehen werden, wenn

(lehnt jedenfalls derogatorisches Gewohnheitsrecht ab); G. SEIDLER, Zur Lehre vom Gewohnheitsrecht auf dem Gebiete des österreichischen Staats- und Verwaltungsrechtes, in: Festschrift zum siebzigsten Geburtstage Sr. Exzellenz Dr. Joseph Unger, Stuttgart I 898, 54I-584 (562); H. Kelsen, Hauptprobleme der Staatsrechtslehre (I9II), unveränderter Nachdruck Tübingen I923, IOI; W. JellineK, Gesetz, Gesetzesanwendung und Zweckmäßigkeitserwägungen, Tübingen
I9I3, 22 f.; G. ANSCHÜTz, in: Preußisches Verwaltungsblatt 22 (I90I) 87 r. Sp. mit Anm. I8; H. NAwIASKy, Zur Lehre vom Gewohnheitsrecht, in: Festschrift zur Feier des fünfzigjährigen Bestehens des Bayerischen Verwaltungsgerichtshofes, München [u. a.] I929, I 56-I 66 (I 59 ff.). Die Anerkennung durch staatliche Organe (Gerichte, Verwaltungsbehörden) lassen immerhin genügen R. SCHMIDT, Einführung in die Rechtswissenschaft, 2. Aufl. Leipzig I923, 53 Anm. I; R. THO-
MA, Der Vorbehalt der Legislative und das Prinzip der Gesetzmäßigkeit von Verwaltung und Rechtsprechung, in: Handbuch des Deutschen Staatsrechts, hg. von G. ANschütz, R. ThомA, 2. Band, Tübingen I932, II, $\mathbb{S} 76,22 \mathrm{Iff}$. (230 Anm. 25).

5 C. G. Bruns, Das heutige Römische Recht, in: Encyklopädie der Rechtswissenschaft in systematischer Verarbeitung, hg. von F. v. Holtzendorff, 4. Aufl. Leipzig I 88 I, 385 ff. (398 f.);

K. Binding, Handbuch des Strafrechts, I. Band, Leipzig I 885 , I9 8 f. mit Anm. 6, 2 IO; G. RÜMELIN, Das Gewohnheitsrecht, in: (Jherings) Jahrbücher für die Dogmatik des heutigen römischen und deutschen Privatrechts 27 (I 889) I 53-252 (I 86 ff., 209); R. STAMMLER, Über die Methode der geschichtlichen Rechtswissenschaft, in: Festgabe zu Bernhard Windscheids fünfzigjährigem Doktorjubiläum, Halle I 888 , I ff., 48 f.; vgl. auch DERs., Lehrbuch der Rechtsphilosophie, 3. Aufl. Berlin, Leipzig I928, I40 (Gewohnheitsrecht und seine Voraussetzungen gehören zum »Inhalte geschichtlich bedingten Rechts «); M. GMÜR, Zivilgesetzbuch und Gewohnheitsrecht, in: Festgabe für Eugen Huber zum siebzigsten Geburtstage, dargebracht von der Juristischen Fakultät der Universität Bern, Bern I9I9, II-52 ( 17 f.); K. v. GAREIs, Rechtsenzyklopädie und Methodologie als Einleitung in die Rechtswissenschaft, 5. Aufl. mit Zusätzen von L. WENGER, Gießen I920, 4I, 52. Die Anerkennung durch staatliche Organe lassen genügen P. OERTMANN, Staatliche und gesellschaftliche Rechtsbildung, in: Archiv für Bürgerliches Recht 40 (I9I4) 709I (80f.); M. E. MAYER, Rechtsphilosophie, Berlin I922, 60.

6 Außer den o. Fn. 4 am Ende Genannten auch z. B. noch KELSEN, Hauptprobleme (Fn. 4) IO2.

7 Eisele, Unverbindl. Gesetzesinhalt (Fn. 3) 2 I.

8 Gierke, Dt. Privatrecht (Fn. 3) I, I6I.

9 In der Reihenfolge der Zitate LABAND, Staatsrecht (Fn. 4) 69; Kelsen, Hauptprobleme (Fn. 4) IOI. 
Io NAwIASKY, Lehre vom Gewohnheitsrecht (Fn. 4) I 59.

I I Vgl. nur etwa Gierke, Dt. Privatrecht (Fn. 3) I, I6I, »immer wird der Strom des Rechtslebens mächtiger sein als er « (der Gesetzgeber); Crome, System (Fn. 3) 85, »Das echte Gewohnheitsrecht hat sich noch immer mit elementarer Kraft durchgesetzt."

I 2 So die von R. Stammler begründete "Rechtsbruchtheorie " (Über die Methode [Fn. 5], 48 f.; Lehrbuch [Fn. 5], I40-I42). Zustimmend B. WindscheID, Lehrbuch des Pandektenrechts, I. Band, 9. Aufl., bearb. von TH. KIPP, Frankfurt am Main I906, 93 f., Anm. 3; wohl auch Rümelin, Bindende Kraft (Fn. 3) 28 (»zur Zeit am meisten verbreitete Lehre «); vgl. auch die Nachweise bei R. Thома, Die Funktionen der Staatsgewalt. I. Grundbegriffe und Grundsätze, in: AnschüTz/THOMA, Handbuch (Fn. 4), 2. Band, \7I, IO8 ff. (I IO Anm. 6).

I3 H. Kelsen, Reine Rechtslehre. Einleitung in die rechtswissenschaftliche Problematik, Leipzig, Wien I934, 64, 67; noch deutlicher in der 2. Aufl. Wien I960, 229, 232 f. Zum Ganzen J. SCHRÖDER, Theorie des Gewohnheitsrechts (Fn. 2) 56I-564.

I4 Eine umfassende Darstellung der Theoriegeschichte scheint nicht $\mathrm{zu}$ existieren. Einige Hinweise zur Lehre im I9. Jahrhundert gibt St. Vogenauer, Die Auslegung von Gesetzen in England und auf dem Kontinent, Tübingen 200I, 556, 567.

I 5 So E. R. Bierling, Juristische Prinzipienlehre, IV, Tübingen I9II, I95.

I 6 Dazu K. R. Sontag, Die Redaktionsversehen des Gesetzgebers, insbesondere auf strafrechtlichem Gebiete, Freiburg i. Br. I874, I2, 33; Binding, Handbuch (Fn. 5) 460 Anm. 9; Bierling, Jur. Prinzipienlehre, IV (Fn. I 5) I96f. Anm. I I.

I7 Th. R. Schütze, Studien zum deutschen Strafgesetzbuche, in: Goltdammers Archiv 20 (I 872)

sie sich auf eine und dieselbe Autorität zurückführen lassen «, und diese Autorität »bezeichnet man gemeinhin als Staat « ${ }^{\circ}{ }^{\circ}$

Auf den ersten Blick scheint zwar die historische Tatsache, dass sich das Gewohnheitsrecht noch immer gegen abweichendes Gesetzesrecht durchsetzen konnte, ${ }^{\text {II }}$ für die zivilrechtliche, "staatsfreie« Lehre zu sprechen. Nach der gegnerischen Gestattungstheorie beweist dieses Phänomen aber nicht die rechtliche Gleichwertigkeit des Gewohnheitsrechts, sondern nur seine (gelegentliche) faktische Überlegenheit. Wenn sich gegen verbietendes Gesetzesrecht ein Gewohnheitsrecht bildet, dann liegt darin eben ein Rechtsbruch, der aber wie eine Revolution neues Recht schaffen kann. ${ }^{{ }^{2} 2}$ Nach Kelsens »Stufenbau «-Lehre ändert sich in diesem Falle (revolutionär) die Grundnorm, die nun auch eine Rechtserzeugung durch Gewohnheitsrecht zulässt. ${ }^{\mathrm{I3}}$ Mit dieser Begründung kann dann zwar das Gewohnheitsrecht auch auf der Grundlage der "Gestattungstheorie « derogierende Kraft bekommen, so dass sich im Ergebnis die Ansichten der Publizisten und der (meisten) Zivilisten annähern. Im Ausgangspunkt stehen sie sich aber unversöhnbar gegenüber.

\section{Das Redaktionsversehen und die Theorie der Gesetzesauslegung}

Unter Redaktionsversehen ${ }^{\mathbf{1 4}}$ versteht man »Textgestaltungen ..., die im Widerspruch mit dem wirklichen Willen der beteiligten Faktoren bei der Redaktion oder Zusammenstellung der gefassten Beschlüsse ... in das Gesetz hineingekommen und dann bei der ganzen weiteren Beratung übersehen, also bloß scheinbar gebilligt worden sind $« .{ }^{{ }^{5}}$ Das heißt, der schließlich beschlossene, sanktionierte Text deckt sich nicht mit dem wirklichen Willen der gesetzgebenden Faktoren (während der bloße Druckfehler den willensentsprechenden und schon sanktionierten Text in einem späteren Stadium, vor allem beim Abdruck im Gesetzblatt, verändert). Ein bekanntes Beispiel aus dem Kaiserreich ist $\mathbb{9} 95$ des Militärstrafgesetzbuchs von I872. ${ }^{16}$ Die Vorschrift betrifft die qualifizierte Verweigerung des Gehorsams, welche nach dem Entwurf der Reichstagskommission mit »Gefängnis oder Festung bis zu 5 Jahren, im Felde Gefängnis oder Festung nicht unter einem Jahre " bedroht sein sollte. Durch ein Versehen fielen im Reichstag

350-373 (353, 356, 373); C. G. v. Wächter, Strafrechtliche Fragen, in: Der Gerichtssaal 29 (I 878 ) 32I-339 (322 f., 338). Zum Teil übereinstimmend, aber mit anderer Begründung SonTAG, Redaktionsversehen (Fn. I6) 26,33; Bierling, Jur. Prinzipienlehre, IV (Fn. I 5 ) I95 f., die gewisse Redaktionsversehen wie Druckfehler behandeln wollen. Gegen berichtigende Auslegung von Redaktionsversehen im Strafrecht aber z. B. F. v. LiszT, Lehrbuch des deutschen Strafrechts, 3. Aufl. Berlin, Leipzig I 888,83 .

I 8 So ausdrücklich Bierling, Jur. Prinzipienlehre, IV (Fn. I 5) I94; L. EnNeCCERUs, Lehrbuch des Bürgerlichen Rechts, I. Band, I. Abteilung, Einleitung, Allgemeiner Teil, 6. bis 8. Aufl., Marburg I9I I, $\mathbb{S} 47$, I08; PH. Heck, Gesetzesauslegung und Interessenjurisprudenz, Tübingen I9I4, I4I, I45, 2 I I. 
die neun Anfangswörter »Gefängnis « bis »im Felde « weg, so dass nun auch Ungehorsam in Friedenszeiten mit der Mindeststrafe von einem Jahr (statt von einem Tag!) zu ahnden gewesen wäre.

In der strafrechtlichen Literatur des Kaiserreichs wird bald die Meinung herrschend, dass der Richter solche Redaktionsversehen selbst korrigieren kann. ${ }^{\mathbf{I 7}}$ Das scheint im Wesentlichen auch die Ansicht der Zivilisten zu sein. Für die »subjektive« Auslegungstheorie, welche die Auslegung am Willen des Gesetzgebers orientiert, handelt es sich um einen klaren Fall der Divergenz zwischen gesetzgeberischem Willen und Wort. ${ }^{\text {I8 }}$ Eine berichtigende Auslegung ist hier ohne weiteres gerechtfertigt. Dementsprechend lässt eine Reihe von subjektivistischen Autoren eine interpretative Korrektur von Redaktionsversehen zu. ${ }^{19}$ Für die »objektive« Theorie, nach der es bei der Auslegung auf den »vernünftigen «, objektiven Gegenwartssinn ankommt, ist die interpretierende Berichtigung von Redaktionsfehlern zwar nicht ganz so selbstverständlich. Eine Korrektur kommt z. B. für Wach nur dann in Frage, wenn das Gesetz etwas enthält, »was vernünftigerweise nach der das Gesetz beherrschenden Logik und seinen sonstigen Vorschriften nicht gesagt werden konnte ${ }^{20}{ }^{20}$ Andere Objektivisten sind jedoch mit der Berichtigung von Redaktionsversehen großzügiger. ${ }^{2 \mathbf{I}}$ Der Fall, dass das Versehen nicht korrigiert werden muss, weil der irrtümlich erklärte Wille vernünftiger ist als der wirkliche, wird ja auch nicht gerade häufig sein. Im Ganzen kann man also wohl sagen, dass die Zivilistik des frühen 20. Jahrhunderts Redaktionsversehen primär durch Auslegung bewältigt.

In der Publizistik zeigt sich ein völlig anderes Bild. Die staatsrechtlichen Lehrbücher behandeln die Redaktionsversehen im Zusammenhang nicht mit der Auslegung, sondern mit der Gesetzgebung. Regelmäßig erscheinen Druckfehler und Redaktionsversehen an der Systemstelle »Gesetzesbeschluss, Ausfertigung und Verkündung «. Erörtert wird, ob für die Korrektur des Redaktionsfehlers eine einfache Berichtigung im Gesetzblatt ausreicht - wie sie häufig, auch in dem oben erwähnten Fall von $\mathbb{9} 95 \mathrm{MilStGB},{ }^{22}$ erfolgte - oder ob ein neues Gesetz erforderlich ist. ${ }^{23}$ Auch die Aufsatzliteratur ${ }^{24}$ beschäftigt sich fast immer nur mit dieser Alternative. Eine Korrektur durch Auslegung wird, wenn überhaupt, nur kurz erwogen und verworfen. Besonders überraschend ist, dass die Staatsrechtler die abweichende zivil- und strafrechtliche Lite-

I9 F. Regelsberger, Pandekten, I. Band, Leipzig I893, I37 f. (wenn sich der Fehler »aus dem Gesetze selbst oder aus dessen Zusammenhang mit einem andern Gesetze ergibt «); F. GÉNY, Méthode d'interprétation et sources en droit privé positif (I899), 2. Aufl., Paris I919, $\mathbb{9} 94,25$ I Anm. 2 (wenn » une erreur certaine « vorliegt); Crome, System, I (Fn. 3) $\int 20$, IO2 f. Anm. 2I (ohne das Wort »Redaktionsversehen «);
EnNeccerus und Heck, wie vorige Fn.; v. Tuhr, Allg. Teil (Fn. 3) 38 Anm. I4I (außer bei unsinnigem Wortlaut); im Ergebnis wohl auch J. BIERMANN, Bürgerliches Recht, I. Band, Allgemeine Lehren und Personenrecht, Berlin I908, 29. Gegen Korrektur oder abändernde Auslegung, jedenfalls im Falle von $\mathbb{9} 95$ Militärstrafgesetzbuch, aber E. HeIlfron, Lehrbuch des Bürgerlichen Rechts auf der Grundlage des Bürgerlichen
Gesetzbuchs, I. Abteilung, Allgemeiner Teil, 2. Aufl. Berlin I900, 37 Anm. 2 (c).

20 So A. WACH, Handbuch des Deutschen Civilprozessrechts, Leipzig I 885, 266 f. Anm. 2 (der in diesem Fall aber nicht von »Redaktionsversehen « spricht; darunter versteht er nur Fehler, die sich erst nach dem Gesetzesbeschluss einschleichen, sie sind nach seiner Meinung »berichtigungsfähig «). Ähnlich BIndING, Handbuch (Fn. 5) 463.

2 I So F. Endemann, Lehrbuch des Bürgerlichen Rechts, I. Band, 9. Aufl., Berlin I903, $\mathbb{S}$ I 2, 50 Anm. 2 ("Sinnauslegung «); K. Hellwig, Lehrbuch des Deutschen Zivilprozessrechts, 2. Band, Leipzig 1907, I 53, bei »offenbare(n), d. h. aus dem Gesetz selbst deutlich hervorgehende(n) Fehler(n)«; L. BRÜTT, Die Kunst der Rechtsanwendung. Zugleich ein Beitrag zur Methodenlehre der Geisteswissenschaften, Berlin I907, I 53 (Redaktionsfehler machen die jeweilige Vorschrift »hinfällig «); R. STAmmLeR, Theorie der Rechtswissenschaft, Halle I9II, 605 .

$22 \mathrm{RGBl}$. I 873 , I38.

23 A. Arndt, Das Staatsrecht des Deutschen Reiches, Berlin I90I; I 85 f.; LABAND, Staatsrecht (Fn. 4) 53 f.; L. DAmbitsch, Die Verfassung des Deutschen Reiches, Berlin I9IO, 52-55; M. v. SEYDEL, Bayerisches Staatsrecht, I, auf der Grundlage der 2. Aufl. neu bearb. von R. Piloty, Tübingen I9I3, 842; G. Meyer, G. AnschütZ, Lehrbuch des Deutschen Staatsrechts, 7. Aufl. München, Leipzig I9I9, $\int$ I 5 8, $667 \mathrm{f}$. In der Zeit der Weimarer Republik ebenso J. Hatscheк, Deutsches und PreuBisches Staatsrecht, II, Berlin I923, IO9-II 2.

24 P. LABAND, Berichtigung von Reichsgesetzen, in: DJZ I903, 30I-304 (303); J. LuKas, Fehler im Gesetzgebungsverfahren, in: Das Recht I I (I907) 669-683. Anders allerdings O. LINDEMANN, Berichtigung der im verkündeten Gesetz enthaltenen Redaktionsversehen, in: AöR I4 (I899) I 45I75. 
25 Selbst in den reichhaltigen, die Literatur des Kaiserreichs zusammenfassenden Literaturhinweisen der letzten Auflage von MeYer / ANSCHÜTZ (Fn. 23) 667 f. erscheint mit seiner abweichenden Ansicht nur der Strafrechtler SonTAG (Fn. I6), während etwa Bierling, Enneccerus, v. Tuhr, Heck und die ältere Literatur nicht erwähnt werden.

26 Soweit ich sehe, weist als einziger Zivilrechtler Heck, Gesetzesauslegung (Fn. I8) I45 Anm. 2 I 8 auf die abweichende Ansicht eines Publizisten (Labands) hin. Er schreibt denn auch vorsichtig, es werde (nur?) "auf dem Gebiete des Privatrechts " die interpretative Korrektur von Redaktionsfehlern für zulässig gehalten.

27 Vgl. die 1932 gemachte Bemerkung von Tнома, Vorbehalt (Fn. 4) 235, die Bearbeitung der Frage nach dem Verhältnis des Richters zum Gesetz dürfe »von der Staatsrechtswissenschaft nicht dauernd den anderen Disziplinen der Rechtswissenschaft allein überlassen bleiben ".

28 In der Reihenfolge der Zitate LABAND, Berichtigung (Fn. 24) 302/ 303; Meyer / AnschüTz, Lehrbuch (Fn. 23) 667. Selbst LINDEMANN, Berichtigung (Fn. 24), der eine richterliche Korrektur zulassen will, meint, es handele sich dabei nicht um Auslegung (I 59).

29 In: Pr. Vbl. 37 (I9I7) 52I f. (Leitsatz, 52 I li. Sp.).

30 Dazu C. Sснотт, »Interpretatio cessat in claris «. Auslegungsfähigkeit und Auslegungsbedürftigkeit in der juristischen Hemeneutik, in: Theorie der Interpretation vom Humanismus bis zur Romantik Rechtswissenschaft, Philosophie, Theologie, hg. von J. SCHRÖDER, Stuttgart 200I, I 55-I89; SCHRÖDER, Recht als Wiss. (Fn. 2) I3 8 .

3I Dagegen sprechen sich auf der Grundlage der "subjektiven " Theorie z. B. Heck, Gesetzesauslegung (Fn. I 8 ) I 2 I ff., I 38 ff. und H. Stoll, Rechtsstaatsidee und Privatrechtslehre, in: JhJb 76 (I926) I34-206 (I69) aus, auf der Grundlage der »objektiven « Theorie z. B. WACH, Handbuch (Fn. 20) 269; BINDING, Handbuch

ratur so gut wie nie überhaupt zur Kenntnis nehmen. ${ }^{25}$ Dasselbe gilt allerdings auch umgekehrt. ${ }^{26}$ Zivilistik und Publizistik scheinen sich in zwei ganz verschiedenen Welten zu bewegen.

Die entscheidende, wenn auch nirgends deutlich ausgesprochene Differenz liegt offenbar in den Vorstellungen von der Gesetzesinterpretation. Das öffentliche Recht hat im frühen 20. Jahrhundert keine eigene Auslegungstheorie entwickelt. ${ }^{27}$ Aber die wenigen Bemerkungen, die im Kontext des Redaktionsfehler-Problems dazu gemacht werden, deuten auf eine enge, strikt am Wortlaut orientierte Interpretationslehre hin. Die Berichtigung von Redaktionsversehen, so heißt es, gehe »weit über die Grenzen der Auslegung hinaus ", und der Richter sei nur berechtigt »zur Klarstellung unklarer Gesetzesvorschriften nach den Regeln grammatischer und juristischer Interpretation $\ll .{ }^{28}$ Das preußische Oberverwaltungsgericht verweigert die Korrektur eines Redaktionsfehlers mit der Begründung, wenn »die Bedeutung der Worte zu Zweifeln keinen Raum « gebe, komme es nur auf den "gemeinverständliche(n) Sinn der Worte « an. ${ }^{29}$ In der Publizistik legt man also die alte, im Laufe der Zeiten immer wieder verworfene und immer wieder neu entdeckte Regel »interpretatio cessat in claris $\aleph^{3 \circ} \mathrm{zu}$ Grunde oder, wenn man so will, das Prinzip vom Wortlaut als Grenze der Auslegung. Dagegen hat sich in der Zivilistik des frühen 20. Jahrhunderts dieses Prinzip nicht wirklich durchgesetzt. ${ }^{3 \mathrm{I}} \mathrm{Ju}$ ristisch-argumentativ entscheiden ließ sich der Streit ebenso wenig wie der über das Gewohnheitsrecht. Bei den Grundsätzen der Gesetzesauslegung gibt es, vielleicht abgesehen von einem kleinen »harten Kern « (Wortsinn, Kontext), kein richtig oder falsch. Sie sind historisch wandelbar und orientieren sich an dem jeweiligen Rechtsbegriff. ${ }^{32}$

\section{Ergebnis}

In zwei rechtstheoretischen Grundfragen (Abhängigkeit des Gewohnheitsrechts vom Staat, Reichweite der Gesetzesauslegung) weichen um I900 die Ansichten der Publizisten und der Zivilisten auffällig voneinander ab. Für die Staats- und Verwaltungsrechtler hat der Staat ein Rechtsschöpfungsmonopol und das Gesetz eine gewisse Stabilität, die seiner interpretativen Veränderung enge Grenzen setzt. Die Publizisten denken offenbar »vom Staat her «, ${ }^{33}$ der ja auch ihr professioneller Gegenstand ist. Dagegen

(Fn. 5) 465 f.; ENDEMANN, Lehrbuch (Fn. 2I) $\mathbb{1}$ I2, 50 Anm. 2; E. Zitelmann, Das Recht des Bürgerlichen Gesetzbuchs, Allgemeiner Teil, Leipzig I900, 4 f.; STAMMLER, Theorie (Fn. 2I) 604; H. ReICHel, Gesetz und Richterspruch, Zürich I9 I 5, 66.

32 In "Recht als Wissenschaft " (Fn. 2) habe ich versucht, diesen Zusammenhang im Einzelnen zu zeigen.
33 Ich übernehme den Ausdruck von F. GÜNTHER, Denken vom Staat her. Die bundesdeutsche Staatsrechtslehre zwischen Dezision und Integration I949-I970, München 2004. Günthers Untersuchung läuft allerdings darauf hinaus, dass dieses Denken "zu Beginn der siebziger Jahre bei der Mehrheit der Staatsrechtslehrer gänzlich in den Hintergrund « getreten sei (32I). 
sind die Zivilisten offenbar gewöhnt, vom Individuum her zu denken, das subjektive Rechte hat, selbst Recht schaffen kann und sich zutraut, auch mit dem staatlichen Gesetz etwas freier umzugehen. - Als in den letzten Jahren meiner Tübinger Lehrtätigkeit einmal ein junger Mann meine Vorlesungen störte (ich habe vergessen, warum), ergriffen ihn zwei hilfsbereite Hörer links und rechts am Arm und geleiteten ihn nach draußen. Ich hielt das für eine erlaubte Nothilfe gegen einen rechtswidrigen Angriff (Hausfriedensbruch, Nötigung). Ein strafrechtlicher Kollege, mit dem ich den Fall erörterte, gab mir zunächst recht, kam dann aber ins Grübeln und konnte sich schließlich nicht entscheiden. Als ich meine öffentlichrechtliche Kollegin fragte, erklärte sie meine Ansicht klipp und klar für falsch. Im öffentlichen Raum gälten besondere Grundsätze, ich hätte die Universitätsverwaltung und die Polizei um Hilfe bitten müssen. Wer hatte recht, oder ist vielleicht beides »vertretbar «? Wie es scheint, wird Rechtsunsicherheit nicht nur durch fehlende oder mangelhafte Gesetze erzeugt, sondern auch durch unterschiedliche professionelle Denkgewohnheiten der Juristen.

Jan Schröder 